\title{
Influence of nitrogen on tunneling barrier heights and effective masses of electrons and holes at lightly-nitrided $\mathrm{SiO}_{2} / \mathrm{Si}$ interface
}

\author{
C. Y. Ng, T. P. Chen, ${ }^{a}$ Y. Liu, and C. Q. Sun \\ School of Electrical and Electronic Engineering, Nanyang Technological University, \\ Singapore 639798 Singapore \\ S. Fung \\ Department of Physics, The University of Hong Kong, Hong Kong
}

(Received 25 May 2004; accepted 16 August 2004)

\begin{abstract}
We have determined both the effective masses and the barrier heights for electrons and holes in pure $\mathrm{SiO}_{2}$ and lightly nitrided oxides with various nitrogen concentrations up to 4.5 at $\%$. In contrast to previous studies which were usually carried out by assuming a value for either the effective mass or the barrier height, this study does not make such an assumption. The approach is proven to be reliable by examining the result for the well-studied pure $\mathrm{SiO}_{2}$ thin films. It is observed that with the increase of the nitrogen concentration the effective masses increase while both the barrier heights and the energy gap decrease. (C) 2004 American Institute of Physics. [DOI: 10.1063/1.1805715]
\end{abstract}

In the modeling and simulation of tunneling current across pure or nitrided $\mathrm{SiO}_{2}$ thin films, both the tunneling barrier height and effective mass for electrons (or holes) at the oxide/Si interface must be known. For electron tunneling from the conduction band, the effective mass $m_{\mathrm{ox}}$ is usually assumed to be $0.5 m_{e}$ (Refs. 1-5 ) or $0.4 m_{e}$ (Ref. 6 ) where $m_{e}$ is the mass of free electrons, while the barrier height is assumed to be $3.15 \mathrm{eV} .^{7}$ Frequently, one of the two parameters (either the effective mass or the barrier height) is obtained from the Fowler-Nordheim (F-N) tunneling current by assuming a value for the other parameter. For example, for electron tunneling, the barrier height $\Phi_{b}$ at $\mathrm{SiO}_{2} / \mathrm{Si}$ interface obtained is $2.9-3.12 \mathrm{eV}$ under the assumption of $m_{\mathrm{ox}}$ $=0.5 m_{e},{ }^{2,4,8}$ and the effective mass obtained under the assumption of $\Phi_{b}=3.15 \mathrm{eV}$ is $0.36 m_{e}{ }^{7}$ The discrepancies in the values of the effective masses and the barrier heights can result in a large difference in the simulation of the tunneling current. On the other hand, it has been reported that the barrier heights decrease with nitrogen concentration in oxynitrides. ${ }^{1,9,15}$ Although the barrier heights are known to be dependent on the nitrogen concentration, the electron effective mass is still assumed to be a constant $\left(0.5 m_{e}\right)$ in the modeling of tunneling current for high nitrogen concentrations. ${ }^{1}$ Obviously such an assumption needs to be justified. For $\mathrm{Si}_{3} \mathrm{~N}_{4} / \mathrm{Si}$ interface, the electron effective mass obtained is $0.4 m_{e}$ using the barrier height of $0.2 \mathrm{eV} ;{ }^{10}$ but the barrier height obtained is $2.12 \mathrm{eV}$ by using the electron effective mass of $0.5 m_{e}{ }^{11}$ These studies were carried out on either oxynitride with high nitrogen concentrations or $\mathrm{Si}_{3} \mathrm{~N}_{4}$ films. Few studies have been reported on the determination of both the effective mass and the barrier height without assuming a value for one of the two parameters for oxynitrides with low nitrogen concentration. Such a study is important because the nitrogen concentration is usually low (a few percentages) for practical metal-oxide-semiconductor (MOS) fabrication and accurate values of the two parameters

${ }^{a)}$ Electronic mail: echentp@ntu.edu.sg are critical to precise simulation of gate oxide tunneling current for nanoscale MOS devices. In this work, we have determined both the effective masses and the barrier heights for electrons and holes without assuming any values for the two parameters for pure $\mathrm{SiO}_{2}$ and lightly nitrided oxides with various nitrogen concentrations up to 4.5 at $\%$. It is found that with the increase of nitrogen concentration the effective masses increase while the barrier heights decrease.

The devices used in this study were $n^{+}$and $p^{+}$ polycrystalline-silicon (poly- Si-gate MOS capacitors i.e., NMOS and PMOS capacitors) with the gate oxide grown on $p$-and $n$-type $\mathrm{Si}$ substrates, respectively. The gate oxide thickness is $6.7 \mathrm{~nm}$, and the device area is $100 \mu \mathrm{m}^{2}$. The gate oxides with different nitrogen concentrations were grown by rapid thermal oxidation followed by in situ rapid thermal nitridation in NO gas ambient. The interfacial nitrogen concentrations $\left(\mathrm{N}_{\text {int }}\right)$ were 1.8, 3.0, 4.0, and 4.5 at $\%$ as determined from secondary ion mass spectroscopy measurement. In addition, a pure $\mathrm{SiO}_{2}$ thin film with the same oxide thickness was also used in this study. The effective oxide thickness was determined from capacitance-voltage $(C-V)$ characteristics by using a quantum mechanical $C-V$ simulator. ${ }^{12}$ Current-voltage $(I-V)$ measurements were carried out at room temperature with Keithley 4200 semiconductor characterization system and $C-V$ measurements were performed with a HP4284A LCR meter at the frequency of $1 \mathrm{MHz}$.

Figure 1 shows the $I-V$ characteristics for the NMOS and PMOS capacitors under positive and negative gate biases for $\mathrm{N}_{\text {int }}=4.5$ at $\%$, respectively. As can been seen in this figure, the F-N tunneling starts as $\sim 8$ and $\sim 9 \mathrm{~V}$ for the NMOS and PMOS capacitors, respectively. Therefore, to study the F-N tunneling in the NMOS and PMOS capacitors, the gate voltage must be larger than the above two values, respectively. For a given gate voltage (either positive or negative), the oxide field $E_{\mathrm{ox}}$ can be calculated by taking into account the flat-band voltage $\left(V_{\mathrm{FB}}\right)$ and the silicon surface band bending as described in Refs. 13 and 14. 


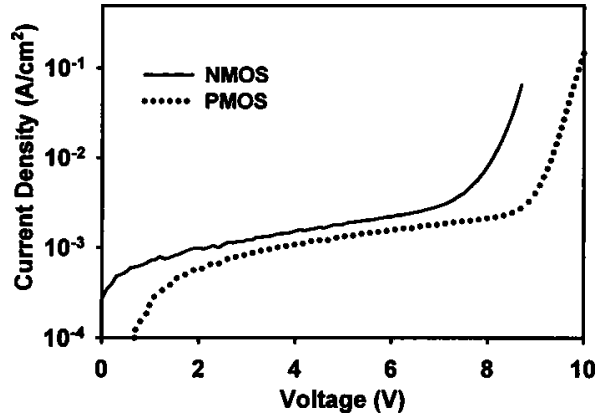

FIG. 1. I-V characteristics for the NMOS and PMOS capacitors with the nitrogen concentration of 4.5 at $\%$ under positive and negative gate biases, respectively. below: ${ }^{7}$

A simple expression for the F-N tunneling is given

$$
J=A E_{\mathrm{ox}}^{2} \exp \left(-B / E_{\mathrm{ox}}\right)
$$

where $J$ is the current density of the F-N tunneling current through the oxide layer, $E_{\mathrm{ox}}$ is the oxide field, and $A$ and $B$ are two constants given by

$$
\begin{aligned}
& A=\frac{q^{3}}{8 \pi h m_{\mathrm{ox}} \Phi_{b}}, \\
& B=\frac{4}{3} \frac{\left(2 m_{\mathrm{ox}}\right)^{1 / 2}}{q \hbar} \Phi_{b}^{3 / 2},
\end{aligned}
$$

where $q$ is the electronic charge, $m_{\mathrm{ox}}$ is the effective mass for electrons (or holes) in the oxide layer, $h$ is the Planck constant, $\hbar$ the reduced Planck constant, and $\Phi_{b}$ the tunneling barrier height for electrons or holes. It should be noted that the above expression for F-N tunneling was derived for a triangular potential barrier under the low-temperature approximation. The image force will modify the triangular potential barrier. ${ }^{17}$ In addition, other effects such as the temperature dependence of the F-N tunneling current, ${ }^{18,21}$ the barrier height variation with the oxide thickness, ${ }^{17,18}$ and the dependence of the effective mass on oxide thickness, ${ }^{19,20}$ may need to be taken into account. Several approximate expressions have been proposed to take into account the temperature dependence ${ }^{17,18,21,22}$ and the image force effect. ${ }^{17}$ In the present study, for simplicity, we use the above simple expression, i.e., Eqs. (1)-(3) for the analysis of the F-N tunneling current (note that both the temperature and the oxide thickness are kept constant). Nevertheless, as discussed below, good accuracy of the above expression is demonstrated by the comparison of the effective mass and the barrier height obtained from this work with those reported in literature for the well-studied pure $\mathrm{SiO}_{2}$ thin films. On the other hand, it should be pointed out that it is also possible to extend the present approach to include the effects mentioned above, but the analysis would be more complicated.

According to Eq. (1), if the current transport across the oxide layer is dominated by F-N tunneling, then a linear relationship between $\operatorname{In}\left(J / E_{\mathrm{ox}}{ }^{2}\right)$ and $\left(E_{\mathrm{ox}}\right)^{-1}$ should be observed, and the intercept with $y$ axis and the slope of the plot of $\operatorname{In}\left(J / E_{\mathrm{ox}}{ }^{2}\right)$ vs $\left(E_{\mathrm{ox}}\right)^{-1}$ can yield the values of $A$ and $B$,
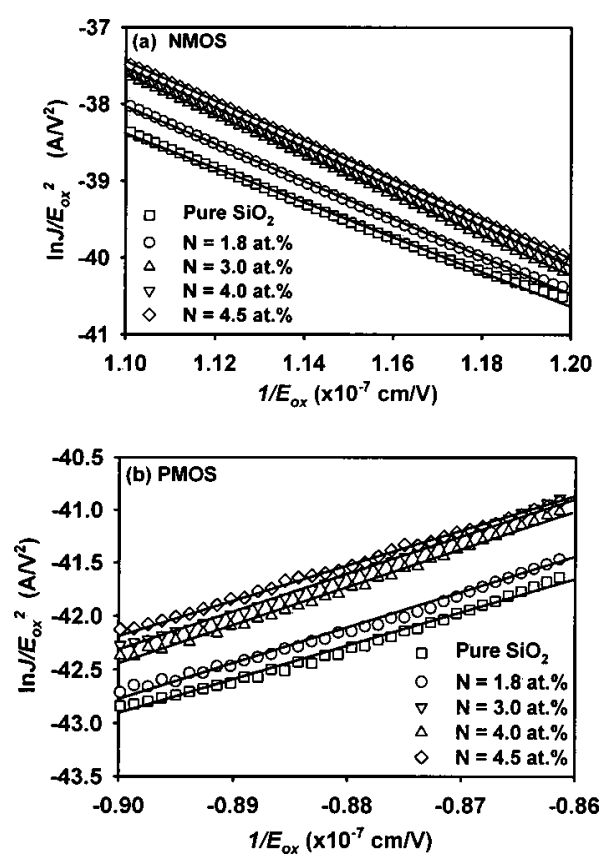

FIG. 2. F-N plots of $\ln \left(J / E_{\mathrm{ox}}{ }^{2}\right)$ vs $1 / E_{\mathrm{ox}}$ as functions of nitrogen concentration for the NMOS (a) and PMOS (b) capacitors.

respectively. Once the values of $A$ and $B$ are obtained, the effective mass and the barrier height can be calculated with Eq. (2) and (3).

Figure 2 shows the plots of $\ln \left(J / E_{\mathrm{ox}}{ }^{2}\right)$ vs $\left(E_{\mathrm{ox}}\right)^{-1}$ as functions of the nitrogen concentration for both the NMOS [Fig. 2(a)] and PMOS [Fig. 2(b)] capacitors. The excellent linear relationships between $\left(J / E_{\mathrm{ox}}{ }^{2}\right)$ and $\left(E_{\mathrm{ox}}\right)^{-1}$ indicate that the current transport is dominated by F-N tunneling. For the NMOS capacitors, electrons tunnel from the Si conduction band at the oxide $\mathrm{Si}$ substrate interface under a positive gate bias; for the PMOS capacitors, holes tunnel from the Si valence band at the interface under a negative gate bias. Note that the capacitors used in this study are actually large MOSFETs with the source and drain grounded and thus there are sufficient minority carriers available in the surface region of the substrate. Using the procedures described above, the effective masses and the tunneling barrier heights for electrons and holes can be determined from the plots of $\ln \left(J / E_{\mathrm{ox}}^{2}\right)$ vs $\left(E_{\mathrm{ox}}\right)^{-1}$. It is worth to mention that in contrast to many other studies, ${ }^{1-4,6-9,11}$ here both the effective masses and the barrier heights are determined without assuming a value for one of the two parameters (i.e., the effective mass and the barrier height). Table I shows the comparison of the effective mass and the barrier height for electrons obtained

TABLE I. Comparison of effective mass and barrier height for electron tunneling from the conduction band at pure $\mathrm{SiO}_{2} / \mathrm{Si}$ interface between this work and previous studies.

\begin{tabular}{lcc}
\hline \hline Effective mass & Barrier height eV & Reference \\
\hline $0.36 m_{e}$ & 3.15 (assumed) & 7 \\
$0.5 m_{e}$ (assumed) & 2.9 & 2 \\
$0.5 m_{e}$ (assumed) & 2.93 & 8 \\
$0.5 m_{e}$ (assumed) & 3.0 & 4 \\
$0.38 m_{e}$ & 3.05 & This work \\
\hline
\end{tabular}




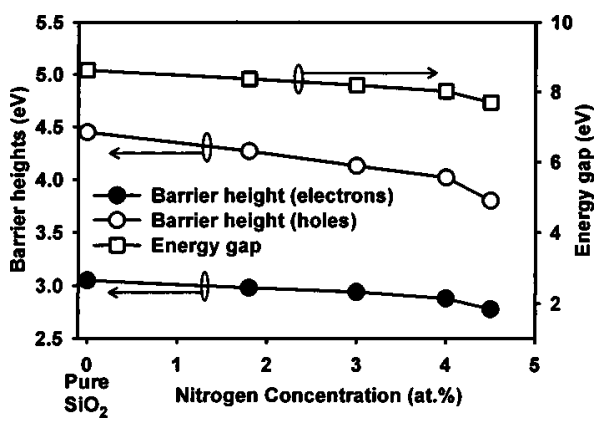

FIG. 3. Dependence of the tunneling barrier heights for electrons and holes as well as the energy gap on the nitrogen concentration.

from this work with those reported in literature $e^{2,4,7,8}$ for the well-studied pure $\mathrm{SiO}_{2}$ thin films. Table I clearly demonstrates the correctness and accuracy of our approach.

The tunneling barrier heights for electrons and holes as functions of the nitrogen concentration are shown in Fig. 3. With increasing the nitrogen concentration, the barrier heights for both electrons and holes decrease. This is consistent with the trend of the reduction of the barrier heights with increasing nitrogen concentration in oxynitrides reported in Ref. 1 and 9. Note that the studies of Refs. 1 and 9 were carried out for high nitrogen concentrations. The reduction of the barrier heights is just a reflection of the reduction of the energy band gap in oxynitrides with nitrogen concentration. ${ }^{9}$ The change of the energy gap with the nitrogen concentration estimated from this study is also shown in Fig. 3. In contrast to the barrier heights, the effective masses for electrons and holes increase with the nitrogen concentration, as shown in Fig. 4. This indicates that the introduction of nitrogen atoms into $\mathrm{SiO}_{2}$ modifies the band curvatures in the energy band structures such that the effective masses increase. Although there are no detailed calculations on the effective masses in oxynidtrides, the calculation of electronic structures has already shown that the effective masses in $\mathrm{Si}_{3} \mathrm{~N}_{4}$ and $\mathrm{Si}_{2} \mathrm{~N}_{2} \mathrm{O}$ are indeed larger than those in $\mathrm{SiO}_{2}{ }^{22}$

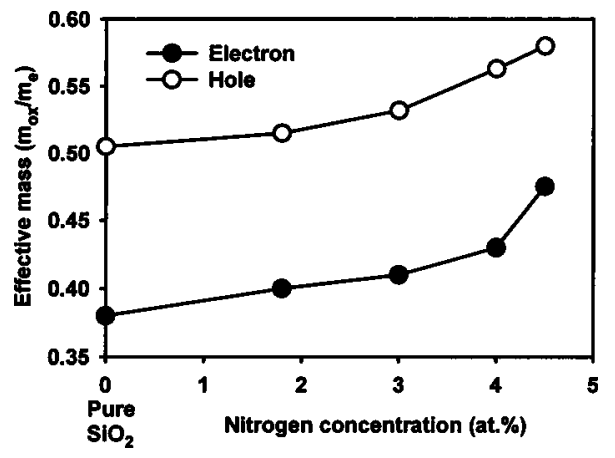

FIG. 4. Dependence of the effective masses for electrons and holes on the nitrogen concentration.
In summary, from the analysis of the F-N tunneling of electrons or holes at the gate oxide Si substrate interface, we have determined both the effective masses and the barrier heights for electrons and holes in pure $\mathrm{SiO}_{2}$ and lightly nitrided oxides with various nitrogen concentrations up to 4.5 at $\%$. In contrast to previous studies reported in literature which were usually carried out by assuming a value for one of the two parameters (i.e., either the effective mass or the barrier height), this study does not make such as assumption. Our approach is proven to be reliable by examining the result for the well-studied pure $\mathrm{SiO}_{2}$ thin films. It is found that with the increase of the nitrogen concentration the effective masses increase while the barrier heights decrease for both electrons and holes.

\section{ACKNOWLEDGMENTS}

This work has been financially supported by the Academic Research Fund from Nanyang Technological University under Project No. RG 8/01. The author would like to thank Chen Ao from Singapore-MIT-Alliance for providing some useful references.

${ }^{1}$ X. Guo and T. P. Ma, IEEE Trans. Electron Devices 19, 207 (1998).

${ }^{2}$ Z. A. Weinberg, J. Appl. Phys. 53, 5052 (1982).

${ }^{3}$ Khairurijal, W. Mizubayashi, S. Miyazaki, and M. Hirose, Appl. Phys. Lett. 77, 3580 (2000).

${ }^{4}$ P. Olivo, J. Sune, and B. Ricco, IEEE Electron Device Lett. 12, 620 (1991).

${ }^{5}$ BSIM 4.3.0 Mosfet Modal, University of California Berkeley (2003).

${ }^{6}$ W. C. Lee, C. Hu, Proc. Symp. VLSI Technology, Dig. Tech. Papers, 2000, p. 198.

${ }^{7}$ M. Depas, B. Vermeire, P. W. Mertens, R. L. Van Meirhaeghe, and M. M. Heyns, Solid-State Electron. 38, 1465 (1995).

${ }^{8}$ M. V. Fischetti, D. J. DiMaria, L. Dori, J. Batey, E. Tierney, and J. Stasiak, Phys. Rev. B 35, 4404 (1987).

${ }^{9}$ H. Yu, Y. -T. Hou, M. F. Li, and D. -L. Kwong, IEEE Trans. Electron Devices 49, 1158 (2002).

${ }^{10}$ V. A. Gritsenko, E. E. Meerson, and Yu. N. Morokov, Phys. Rev. B 57, R2081 (1998).

${ }^{11}$ Y. Shi, X. Wang, and T. P. Ma, IEEE Trans. Electron Devices 46, 362 (1999).

${ }^{12}$ K. Yang, Y. King, and C. Hu, Proc. Symp. VLSI Tech. 1999, p. 77.

${ }^{13}$ T. P. Chen, S. Li, S. Fung, and K. F. Lo, IEEE Trans. Electron Devices 45, 1920 (1998).

${ }^{14}$ Y. Hokari, IEEE Trans. Electron Devices 35, 1299 (1998).

${ }^{15}$ D. M. Brown, P. V. Gary, F. K. Heumann, H. R. Philipp, and E. A. Taft, J. Electrochem. Soc. 115, 311 (1968).

${ }^{16}$ Y. -N. Xu and W. Y. Ching, Phys. Rev. B 51, 17379 (1995).

${ }^{17}$ A. Hadjadj, G. Salace, and C. Petit, J. Appl. Phys. 89, 7994 (2001).

${ }^{18}$ G. Pananakakis, G. Ghibaudo, R. Kies, and G. Papadas, J. Appl. Phys. 78, 2635 (1995).

${ }^{19}$ S. Horiguchi and H. Yoshino, J. Appl. Phys. 58, 1597 (1985).

${ }^{20}$ R. Ludeke, E. Cartier, and A. Schenk, Appl. Phys. Lett. 75, 1407 (1999).

${ }^{21}$ G. Salace, A. Hadjadj, C. Petit, and M. Jourdain, J. Appl. Phys. 85, 7768 (1999).

${ }^{22}$ J. Suné, M. Lanzoni, and P. Olivo, IEEE Trans. Electron Devices 40, 1017 (1993). 